\title{
Efeitos da infusão contínua de cetamina sobre a pressão intra-ocular em cães hipovolêmicos anestesiados com desflurano
}

\author{
[Effects of continuous infusion of ketamine on intraocular pressure in hypovolemic \\ dogs during desflurane anesthesia] \\ C.S. Honsho $^{1}$, I.C. Talieri ${ }^{1}$, N. Nunes ${ }^{2}$, J.C.D. Moreno ${ }^{1}$, A.P. Souza ${ }^{1}$ \\ ${ }^{1}$ Doutorando em Cirurgia Veterinária - FCAVJ - UNESP - Jaboticabal, SP \\ ${ }^{2}$ Faculdade de Ciências Agrárias e Veterinária - UNESP \\ Rod. Paulo Donatto Castelane s/n \\ 14884-900 - Jaboticabal, SP
}

\begin{abstract}
RESUMO
Compararam-se os efeitos de duas doses de cetamina, administradas em infusão contínua, sobre a pressão intra-ocular (PIO) de 18 cães submetidos à hipovolemia e à anestesia com desflurano. Promoveu-se a hipovolemia em todos os cães, retirando-se $40 \mathrm{ml}$ de sangue $/ \mathrm{kg}$ de peso. A anestesia foi induzida com desflurano, através de máscara facial, até que a intubação orotraqueal fosse permitida. Decorridos 30 minutos, para estabilização dos parâmetros, iniciou-se a infusão contínua de cetamina. Os cães foram distribuídos, aleatoriamente, em três grupos $(n=6)$. O grupo I (controle) recebeu solução salina estéril; o grupo II (GII) recebeu cetamina, na dose de $100 \mu \mathrm{g} / \mathrm{kg} / \mathrm{min}$, e o grupo III (GIII), cetamina na dose de $200 \mu \mathrm{g} / \mathrm{kg} / \mathrm{min}$. A PIO foi medida por tonometria de aplanação. Foram mensurados freqüência cardíaca (FC), ritmo cardíaco, pressão arterial média (PAM), débito cardíaco (DC), pressão venosa central (PVC) e pressão parcial de $\mathrm{CO}_{2}$ no final da expiração $\left(\mathrm{ETCO}_{2}\right)$. $\mathrm{O}$ desflurano não influenciou os resultados da PIO, porém observou-se discreta ação da cetamina em todos os grupos. Foi possível estabelecer relação direta entre os valores de $\mathrm{PIO}$ e de $\mathrm{ETCO}_{2}$. A PIO apresentou relação direta somente com a $\mathrm{ETCO}_{2}$.
\end{abstract}

Palavras-chave: desflurano, cetamina, hipovolemia, pressão intra-ocular, anestesia

\begin{abstract}
Effects of two dosages of continuous infusion of ketamine on the intraocular pressure (IOP) of 18 dogs, subjected to hypovolemia and anesthesia with desflurane were studied. Hypovolemia was induced in all dogs by withdrawal of $40 \mathrm{ml}$ of blood/kg of body weight. Initially, anesthesia was induced through face mask and then by tracheal intubation. After a delay of 30 minutes, for parameters stabilization, continuous infusion of ketamine was initiated. Dogs were randomly allotted in three groups $(n=6)$ : group I (control group) received continuous infusion of saline solution, group II received $100 \mu \mathrm{g} / \mathrm{kg} / \mathrm{min}$ of ketamine and group III received $200 \mu \mathrm{g} / \mathrm{kg} /$ min of ketamine. IOP was measured by applanation tonometry. It was not possible to establish direct correlation between alterations of the mean arterial pressure and central venous pressure to those obtained to intraocular pressure. Desflurane did not affected values of intraocular pressure, however, direct action of ketamine was observed in all groups. It was possible to establish direct correlation between values of intraocular pressure and values of exhaled $\mathrm{CO}_{2}$.
\end{abstract}

Keywords: desflurane, ketamine, hypovolemia, intraocular pressure, anesthesia

Recebido para publicação em 6 de maio de 2003

Recebido para publicação, após modificações, em 4 de fevereiro de 2004

E-mail: newton@fcav.unesp.br 


\section{INTRODUÇÃO}

O controle da pressão intra-ocular (PIO), em níveis abaixo dos limites normais, é reconhecido como essencial para boa anestesia em cirurgias intra-oculares, visto que flutuações na PIO podem ter efeitos catastróficos durante e após tais cirurgias (Cunnigham e Barry, 1986; Collins et al., 1995; Nunes e Laus, 1995).

A PIO é definida como a pressão exercida pelo conteúdo intra-ocular contra a parede que o contém (Murphy, 1985; Cunnigham e Barry, 1986), sendo determinada pela taxa de produção e drenagem de humor aquoso (HA), pelo volume do vítreo, volume sangüíneo da coróide, rigidez da esclera, tensão do músculo orbicularis oculi e pela pressão externa (Duncalf e Weitzener, 1963; Brunson, 1980; Murphy, 1985; Cunnigham e Barry, 1986). A compressão externa do globo, por meio da contração dos músculos extraoculares, pode aumentar diretamente a PIO, ou ter efeitos indiretos, induzindo alterações nos volumes dos componentes intra-oculares (Brunson, 1980; Murphy, 1985).

O HA é um fluido transparente que ocupa as câmaras anterior e posterior e se assemelha a um ultrafiltrado do plasma. A taxa de formação, em condições normais, iguala-se à de drenagem, determinando a PIO com pequena variação diurna (Cunnigham e Barry, 1986; Collins et al., 1995). Sua drenagem ocorre por duas vias: a principal, feita através do ângulo iridocorneano, e a secundária, em menor proporção, através dos espaços intersticiais do músculo ciliar em direção à esclera (Murphy, 1985; Collins et al., 1995).

$\mathrm{O}$ volume sangüíneo intra-ocular depende do balanço entre o influxo e o efluxo de sangue, além do grau de constrição dos vasos sangüíneos intra-oculares. Como outros órgãos, o olho é capaz de auto-regular seu suprimento sangüíneo. Dessa forma, há discreta correlação entre as alterações na pressão arterial sistêmica (PA) e a PIO (Murphy, 1985; Collins et al., 1995).

Em estado de equilíbrio, outro fator que altera o volume sangüíneo intra-ocular é o tônus dos vasos intra-oculares, o qual é influenciado pela pressão arterial de dióxido de carbono $\left(\mathrm{PaCO}_{2}\right)$ (Murphy, 1985; Trim et al., 1985). Segundo Cunnigham (1993), a $\mathrm{PaCO}_{2}$ pode ser mensurada mediante pressão alveolar de $\mathrm{CO}_{2}$ expirado $\left(\mathrm{ETCO}_{2}\right)$ e pelo controle central do diencéfalo.

Fármacos administrados durante a anestesia podem afetar a PIO, pela ação direta sobre o diencéfalo, facilitando ou inibindo a produção do $\mathrm{HA}$, relaxando ou contraindo o músculo orbicularis e os músculos extra-oculares, ou ainda, indiretamente, por meio dos efeitos sobre os sistemas cardiovascular e respiratório (Brunson, 1980; Murphy, 1985; Cunnigham e Barry, 1986; Collins et al. 1995). Jantzen (1988), citado por Sator et al. (1998), e Cunnigham e Barry (1986) enfatizam a importância dos anestésicos nas cirurgias oftálmicas, além de realçarem a redução da PIO com uso de agentes intravenosos e inalatórios, destacando a cetamina como notável exceção.

Dentre as principais características do desflurano, incluem-se: a alta pressão de vapor saturado; o baixo ponto de ebulição (McMurphy e Hodgson, 1995) e a baixa solubilidade em gordura, tornando-o um anestésico de rápida indução e recuperação; e a alteração da profundidade da inconsciência em reposta às mudanças na concentração inspirada (McMurphy e Hodgson, 1995; Clarke et al., 1996; Sator et al., 1998).

A cetamina, um anestésico dissociativo, possui ação simpatomimética e estimula a freqüência cardíaca (FC), resultando no aumento do débito cardíaco. Exerce vasoconstrição periférica que eleva, consideravelmente, a pressão arterial. Entretanto, não altera a pressão venosa. É indicada em casos de choques, especialmente o hemorrágico. A cetamina atua, ainda, aumentando o tônus muscular (Massone, 1994), bem como o dos músculos extra-oculares (Brunson, 1980).

O objetivo deste estudo foi o de observar o comportamento da pressão intra-ocular, segundo os efeitos cardiovasculares e hemodinâmicos induzidos pela infusão contínua de cetamina, durante a anestesia geral com desflurano, em cães submetidos à hipovolemia experimental.

\section{MATERIAL E MÉTODO}

Utilizaram-se 18 cães hígidos, sem raça definida, machos e fêmeas, adultos com pesos entre $10 \mathrm{e}$ 
$15 \mathrm{~kg}$. Os animais foram selecionados segundo a avaliação oftalmológica e hematimétrica de rotina. Foram dispostos, aleatoriamente, em três grupos experimentais: GI - infusão de solução salina; GII - infusão de cetamina na dose de $100 \mu \mathrm{g} / \mathrm{kg} / \mathrm{min}$; e GIII - infusão de cetamina na dose de $200 \mu \mathrm{g} / \mathrm{kg} / \mathrm{min}$.

No dia anterior à experimentação, os cães foram anestesiados com propofol $^{1}(5 \mathrm{mg} / \mathrm{kg})$ por via intravenosa e, após tricotomia e anti-sepsia da face medial do membro pélvico, fez-se a abordagem cirúrgica para cateterização da artéria femoral. O cateter foi ocultado no espaço subcutâneo e a incisão foi fechada com fio de náilon, em pontos simples interrompidos.

Após 24 horas, com os animais sob contenção física, o cateter intra-arterial foi exteriorizado e conectado ao transdutor do equipamento multiparamétrico ${ }^{2}$, para auferição da pressão arterial. $\mathrm{Na}$ entrada da artéria pulmonar, por meio da veia femoral, posicionou-se um cateter de Swan Ganz 5F ${ }^{3}$, guiado pela leitura da onda de pulso observada no monitor do equipamento multiparamétrico.

Realizada a instrumentalização, fez-se a remoção sangüínea $(40 \mathrm{ml} / \mathrm{kg})$, pelo cateter colocado na artéria femoral, com velocidade não superior a $100 \mathrm{ml} / 5 \mathrm{~min}$. O sangue removido foi armazenado em bolsas para transfusão sangüínea e mantido em temperatura ambiente, até a sua reinfusão.

Decorridos 15 minutos, realizou-se a indução anestésica com desflurano ${ }^{4}$, administrado por máscara facial, com fluxo diluente de oxigênio de $41 / \mathrm{min}$. Após intubação orotraqueal, com sonda de Magil, a manutenção foi feita com 1,5 CAM (aferida pela leitura no analisador de gases anestésicos ${ }^{5}$ ) do anestésico diluído em $100 \%$ de oxigênio, com fluxo de $30 \mathrm{ml} / \mathrm{kg}$, administrados em circuito anestésico semi-fechado ${ }^{6}$, dotado de vaporizador específico para o agente anestésico empregado.

\footnotetext{
${ }^{1}$ Propofol - Cristállia produtos químicos farmacêuticos Ltda

${ }^{2}$ Dixtal 2010 - Dixtal Biomédica, Manaus. AM.

${ }^{3}$ Swan-Ganz, Baxter Healthcare 5F Corp. Irvine, CA

${ }^{4}$ Suprane, Baxter Farmacêutica, São Paulo - SP, Brasil

${ }^{5}$ Ohmeda - Mod. 5250 RGM - Processo FAPESP 96/12830-0

${ }^{6}$ Ohmeda - Mod. Excel 210 SE - Processo FAPESP 97/10668-4
}

Trinta minutos após a indução anestésica, deu-se início à infusão contínua de solução salina $0,9 \%$ aos animais, por meio de bomba de infusão ${ }^{7}$. O volume final de infusão foi ajustado para $5 \mathrm{ml} / \mathrm{kg} /$ hora.

Os animais permaneceram em decúbito lateral esquerdo durante todo o período do experimento.

A FC e o ritmo cardíaco foram avaliados pelos traçados eletrocardiográficos (ECG), registrados por meio de eletrocardiografia computadorizada. A pressão arterial média (PAM) foi mensurada pela leitura da onda de pulso (obtida por meio do cateter na artéria femoral), no monitor do equipamento multiparamétrico.

O débito cardíaco (DC) foi auferido pela técnica de termodiluição descrita por NUNES (2002). A pressão venosa central (PVC) foi avaliada por meio de cateter posicionado na veia cava, sendo a leitura auferida em monitor multiparamétrico digital.

A freqüência respiratória (FR) e a $\mathrm{ETCO}_{2}$ foram obtidas pela leitura do aparelho de ventilometria ${ }^{8}$ e do analisador de gases, respectivamente.

As mensurações das PIO, com tonômetro de aplanação ${ }^{9}$, foram realizadas nas córneas dessensibilizadas pela utilização de colírio de cloridrato de proximetacaína ${ }^{10}$. Os cães, inicialmente mantidos em decúbito lateral esquerdo, foram posicionados em decúbito esternal, por ocasião das aferições das pressões intra-oculares dos olhos direito e esquerdo.

Os parâmetros de avaliação foram registrados, após a instrumentalização e antes de qualquer outro procedimento (T0), 15 minutos depois da indução da hipovolemia experimental (T45), decorridos 30 minutos da indução anestésica (T75), cinco minutos após o início da infusão contínua (T80) e aos 15 (T95), 30 (T110) e 45 (T125) minutos, após o começo da infusão contínua.

\footnotetext{
7 Lifemed. Mod. FARS 600

8 Takaoka mod.Ventcare - Processo FAPESP 98/03153-0

9 Tono Pen XL - Mentor Medical Systems

10 Anestalcon - Alcon Laboratórios de Brasil Ltda.
} 
As análises estatísticas foram realizadas segundo HOEL (1974), com o auxílio do programa InStat $^{11}$. Para detectar diferenças entre os momentos, dentro de cada grupo ao longo do tempo, foi utilizada a análise de variância de uma via para repetições múltiplas, seguida pelo teste
Tukey e pelo teste $t$, para comparação de médias entre os grupos em cada momento de avaliação.

Os valores foram expressos em média \pm desvio padrão e considerados significantes quando $\mathrm{P}<0,05$.

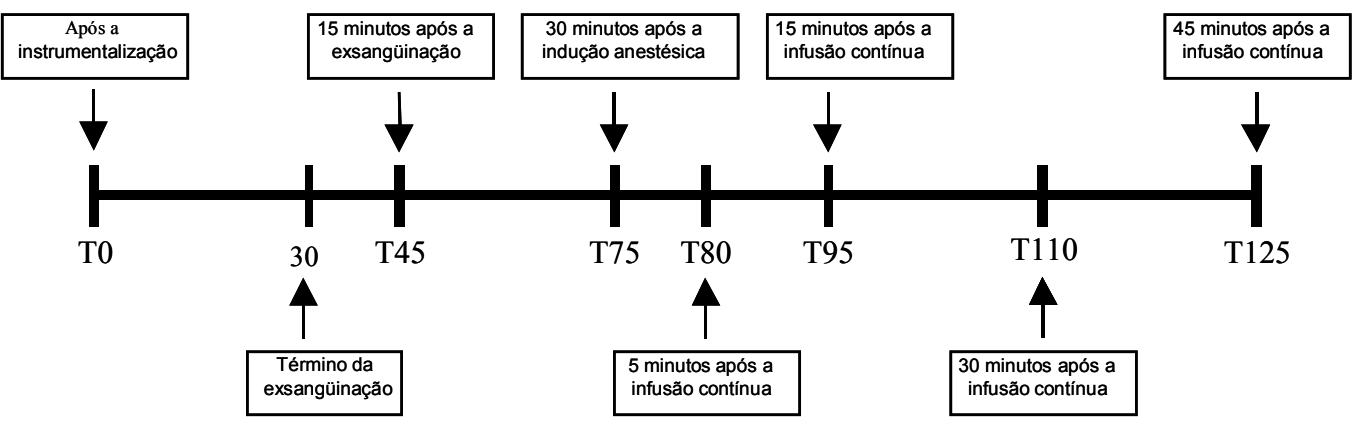

\section{RESULTADOS E DISCUSSÃO}

Não se observaram alterações significativas na PIO dos olhos direito e esquerdo, com os animais mantidos em decúbito lateral esquerdo (Fig. 1; Tab. 1). Entretanto, há relatos sobre a influência da posição corporal na PIO (Hvidberg et al., 1981)

Com a indução da hipovolemia, há diminuição da pressão venosa no lado direito do coração e diminuição da pressão no átrio direito, levando à diminuição dos volumes diastólico e sistólico do ventrículo direito. Assim, há diminuição do volume sistólico do ventrículo esquerdo e, conseqüentemente, do débito cardíaco, ocasionando queda na pressão arterial sistêmica (Cunnigham, 1993). Dessa forma, foi observado, em todos os grupos, aumento compensatório da $\mathrm{FC}$, correspondente à diminuição da $\mathrm{PVC}$ e da PAM, na tentativa de se manter o DC e a perfusão tecidual.

Após 30 minutos da indução anestésica (T75), observou-se aumento da FC em GI. Quanto à PAM, não foram observadas alterações entre grupos. Entretanto, no período entre a exsanguinação (T45) e a indução anestésica (T75), houve diferenças significativas. Quando

${ }^{11}$ GraphPad InStat. Co. da comparação entre grupos e momentos, não se observaram diferenças na PVC. Em relação ao DC, não houve diferença entre os grupos, exceto em T45. Na $\mathrm{ETCO}_{2}$, após a hipovolemia (T45), houve alterações significativas entre os grupos, e alterações em GI e GII, após a indução anestésica (T75) e a infusão contínua de cetamina (T80). Da comparação entre grupos, pôde-se observar alterações entre GI e GII e GI e GIII, após a indução de cetamina (T80). Não houve diferença entre os grupos que receberam infusão de cetamina e verificou-se diferença na FR entre GI e GIII, após a indução de cetamina (T80). Devido à baixa perfusão instituída com a hipovolemia (diminuição do $\mathrm{ETCO}_{2}$ ), houve aumento compensatório da FR.

Vários são os trabalhos na literatura que afirmaram não haver relação entre PA e PIO. Michelson et al. (1994) observaram, no homem, que a PA elevada não alterava a PIO, graças ao aumento na resistência vascular e à constância na velocidade média de fluxo sangüíneo na artéria oftálmica e nas artérias ciliares. Macri (1961a) observou, em gatos, que a pressão nas artérias ciliares não estava associada à pressão sangüínea sistêmica, e que havia dissociação entre as artérias ciliares e a artéria oftálmica, visto que a pressão da artéria oftálmica mantinha íntima relação com a PA. Quando em níveis 
incompatíveis com a perfusão tecidual adequada, a PA pode deprimir a formação do HA (Murphy, 1985). Jantzen et al. (1992) observaram, em suínos, que a hipotensão arterial induzida por drogas não alterava a PIO. Neste estudo, na PAM houve acentuada redução durante a hipovolemia. Essa redução exacerbou-se com o uso do desflurano e manteve-se ao longo do experimento. A PIO apresentou discreta diminuição durante a indução da hipovolemia, mas essa diminuição não se manteve durante a anestesia com desflurano. Dessa forma, não foi possível correlacionar a redução da PAM com os valores obtidos da PIO.
OD

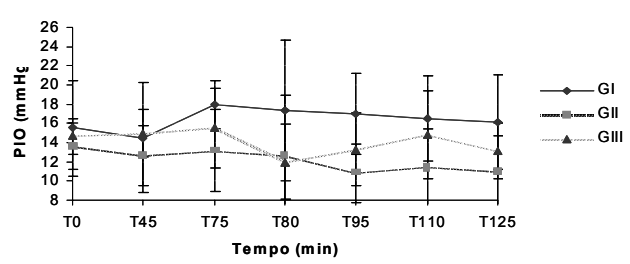

FC

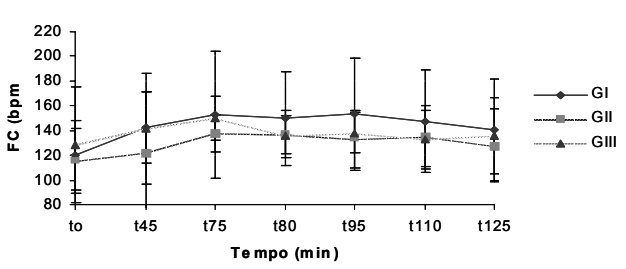

PVC

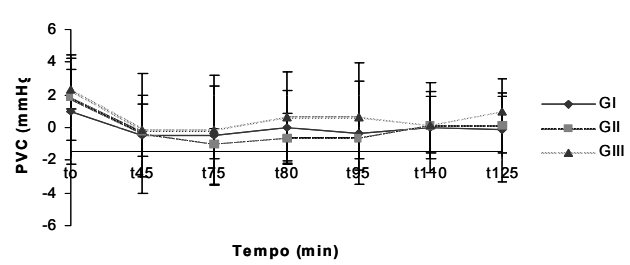

ETCO2

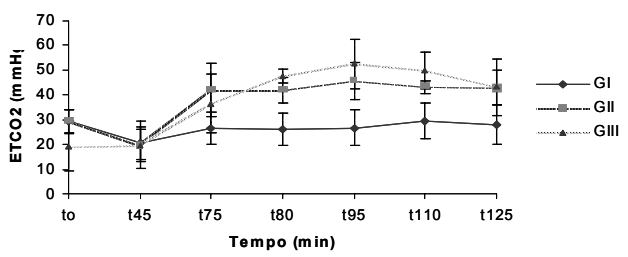

OE
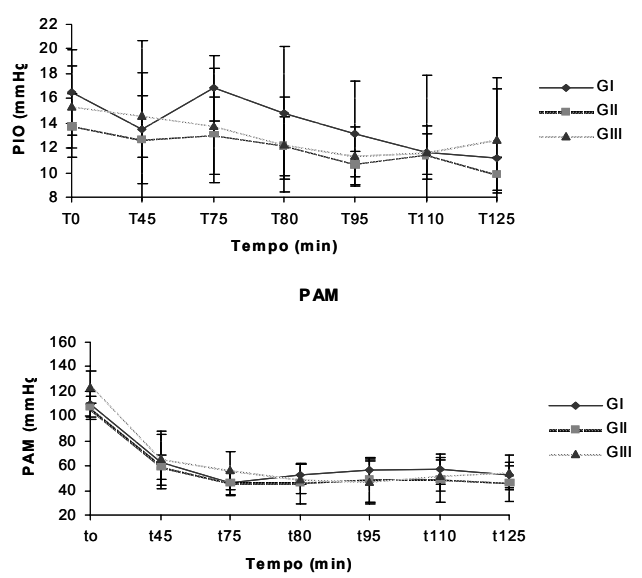

DC

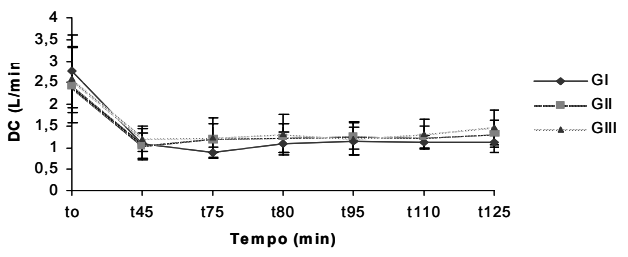

FR

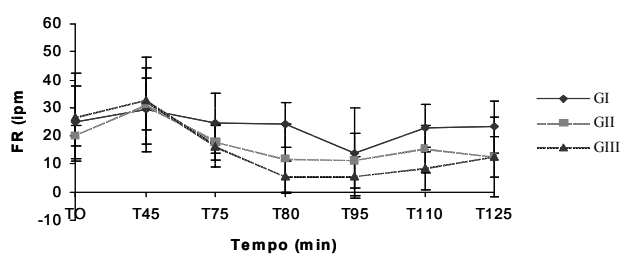

Figura 1. Valores médios de pressão intra-ocular nos olhos direito (PIOD) e esquerdo (PIOE); freqüência cardíaca (FC); pressão arterial média (PAM); pressão venosa central (PVC); débito cardíaco (DC); pressão parcial de $\mathrm{CO} 2$ no final da expiração (ETCO2) e freqüência respiratória (FR) em cães hipovolêmicos, anestesiados com desflurano e infusão contínua de solução salina (GI), de cetamina na dose de $100 \mu \mathrm{g} / \mathrm{kg} / \mathrm{min}$ (GII) e de cetamina na dose de $200 \mu \mathrm{g} / \mathrm{kg} / \mathrm{min}$ (GIII). 
Tabela 1. Valores médios e desvios-padrão de pressão intra-ocular nos olhos direito (PIOD) e esquerdo (PIOE), de freqüência cardíaca (FC), de pressão arterial média (PAM), de pressão venosa central (PVC), de débito cardíaco (DC), de pressão parcial de $\mathrm{CO}_{2}$ no final da expiração $\left(\mathrm{ETCO}_{2}\right)$ e da freqüência respiratória (FR) em cães hipovolêmicos, anestesiados com desflurano e infusão contínua de solução salina (GI), de cetamina na dose de $100 \mu \mathrm{g} / \mathrm{kg} / \mathrm{min}$ (GII) e de cetamina na dose de $200 \mu \mathrm{g} / \mathrm{kg} / \mathrm{min}$ (GIII), de acordo com os momentos

\begin{tabular}{|c|c|c|c|c|c|c|c|c|}
\hline & & T 0 & T 45 & T 75 & T 80 & Т 95 & T 110 & T 125 \\
\hline \multirow{3}{*}{$\begin{array}{l}\text { PIOD } \\
(\mathrm{mmHg})\end{array}$} & $G I$ & $15,5 \pm 4,93$ & $14,5 \pm 5,68$ & $18 \pm 2,53$ & $17,33 \pm 7,31$ & $17 \pm 4,24$ & $16,5 \pm 4,46$ & $16,5 \pm 4,93$ \\
\hline & $G I I$ & $13,67 \pm 2,42$ & $12,67 \pm 3,08$ & $13,17 \pm 4,26$ & $12,67 \pm 6,28$ & $10,83 \pm 3,06$ & $11,5 \pm 3,89$ & $11 \pm 3,69$ \\
\hline & GIII & $14,67 \pm 1,86$ & $15 \pm 2,45$ & $15,5 \pm 4,18$ & $12 \pm 3,90$ & $12,33 \pm 3,78$ & $14,83 \pm 4,58$ & $13,17 \pm 2,93$ \\
\hline \multirow{3}{*}{$\begin{array}{l}\text { PIOE } \\
(\mathrm{mmHg})\end{array}$} & $G I$ & $16,5 \pm 3,45$ & $13,5 \pm 7,23$ & $16,83 \pm 2,64$ & $14,83 \pm 5,38$ & $13,17 \pm 4,26$ & $16,67 \pm 6,19$ & $11 \pm 6,36$ \\
\hline & GII & $13,83 \pm 2,56$ & $12,67 \pm 3,56$ & $13 \pm 3,16$ & $12,17 \pm 2,40$ & $10,67 \pm 1,03$ & $11,5 \pm 1,64$ & $9,83 \pm 1,47$ \\
\hline & GIII & $15,33 \pm 3,33$ & $14,67 \pm 3,39$ & $13,83 \pm 4,58$ & $12,33 \pm 3,83$ & $11,33 \pm 2,34$ & $11,67 \pm 2,16$ & $12,67 \pm 4,13$ \\
\hline \multirow{3}{*}{$\begin{array}{l}\mathrm{FC} \\
\text { (bmp) }\end{array}$} & $G I$ & $120,17 \pm 28,01$ & $142,17 \pm 29,01$ & $152,83 \pm 51,00$ & $149,67 \pm 37,71$ & $153,5 \pm 45,33$ & $147,5 \pm 41,61$ & $140,5 \pm 41,12$ \\
\hline & GII & $115,5 \pm 26,42$ & $121,67 \pm 49,58$ & $137,67 \pm 15$ & $137,33 \pm 19,44$ & $133,1667 \pm 23,7$ & $135,33 \pm 24,83$ & $127,67 \pm 29,33$ \\
\hline & GIII & $128,67 \pm 46,74$ & $141,5 \pm 44,76$ & $150 \pm 18,14$ & $136,33 \pm 15,37$ & $138,17 \pm 16,22$ & $132,83 \pm 24,14$ & $135,83 \pm 30,71$ \\
\hline \multirow{3}{*}{$\begin{array}{l}\text { PAM } \\
(\mathrm{mmHg})\end{array}$} & $G I$ & $109,33 \pm 10,25$ & $63,17 \pm 21,91$ & $46 \pm 9,25$ & $53 \pm 8,00$ & $56,83 \pm 9,02$ & $57,5 \pm 12,42$ & $53 \pm 10,18$ \\
\hline & GII & $106,83 \pm 9,15$ & $58,83 \pm 9,50$ & $45,83 \pm 9,60$ & $45,67 \pm 16,37$ & $48,67 \pm 18,28$ & $48,5 \pm 18,12$ & $45,67 \pm 14,79$ \\
\hline & GIII & $123,5 \pm 13,00$ & $65,83 \pm 21,99$ & $56,17 \pm 15,48$ & $49,17 \pm 11,86$ & $46,83 \pm 17,24$ & $52 \pm 12,51$ & $54,67 \pm 13,76$ \\
\hline \multirow{3}{*}{$\begin{array}{l}\text { PVC } \\
(\mathrm{mmHg})\end{array}$} & $G I$ & $1 \pm 3,22$ & $-0,5 \pm 2,51$ & $-0,5 \pm 3,02$ & $0 \pm 2,28$ & $-0,3 \pm 3,14$ & $0 \pm 2,76$ & $-0,17 \pm 3,13$ \\
\hline & GII & $1,83 \pm 2,64$ & $-0,33 \pm 3,67$ & $-1 \pm 0,89$ & $-0,67 \pm 1,51$ & $-0,67 \pm 1,21$ & $0,17 \pm 2,04$ & $0,17 \pm 1,72$ \\
\hline & GIII & $2,33 \pm 1,21$ & $-0,17 \pm 1,60$ & $-0,17 \pm 3,31$ & $0,67 \pm 2,73$ & $0,67 \pm 2,27$ & $0,17 \pm 1,72$ & $1 \pm 1,10$ \\
\hline \multirow{3}{*}{$\begin{array}{l}\mathrm{DC} \\
(\mathrm{L} / \mathrm{min})\end{array}$} & $G I$ & $2,77 \pm 0,08$ & $1,10 \pm 0,35$ & $0,88 \pm 0,14$ & $1,09 \pm 0,28$ & $1,15 \pm 0,32$ & $1,12 \pm 0,16$ & $1,12 \pm 0,23$ \\
\hline & GII & $2,44 \pm 0,88$ & $1,03 \pm 0,32$ & $1,21 \pm 0,33$ & $1,22 \pm 0,33$ & $1,26 \pm 0,31$ & $1,24 \pm 0,25$ & $1,31 \pm 0,31$ \\
\hline & GIII & $2,58 \pm 0,75$ & $1,20 \pm 0,30$ & $1,22 \pm 0,45$ & $1,32 \pm 0,43$ & $1,21 \pm 0,38$ & $1,31 \pm 0,34$ & $1,47 \pm 0,40$ \\
\hline \multirow{3}{*}{$\begin{array}{l}\mathrm{ETCO}_{2} \\
(\mathrm{mmHg})\end{array}$} & $G I$ & $29,5 \pm 4,55$ & $20,67 \pm 6,62$ & $26,5 \pm 6,47$ & $26 \pm 6,51$ & $26,83 \pm 7,17$ & $29,5 \pm 7,23$ & $27,83 \pm 7,98$ \\
\hline & GII & $29,33 \pm 4,89$ & $19,67 \pm 6,53$ & $42,17 \pm 10,74$ & $42 \pm 5,14$ & $45,83 \pm 7,39$ & $43,17 \pm 2,71$ & $42,83 \pm 7,00$ \\
\hline & GIII & $29,33 \pm 10,21$ & $19,67 \pm 9,56$ & $36,5 \pm 11,93$ & $47,67 \pm 2,66$ & $52,67 \pm 10,07$ & $49,83 \pm 7,47$ & $43,17 \pm 11,29$ \\
\hline \multirow{3}{*}{$\begin{array}{l}\text { FR } \\
\text { (ipm) }\end{array}$} & $G I$ & $24,83 \pm 12,95$ & $29,33 \pm 15,23$ & $24,67 \pm 10,63$ & $24 \pm 8,17$ & $13,83 \pm 16,08$ & $22,83 \pm 8,54$ & $23,17 \pm 9,39$ \\
\hline & GII & $20,17 \pm 3,76$ & $31,33 \pm 9,14$ & $17,83 \pm 6,37$ & $11,67 \pm 12,40$ & $11,33 \pm 9,71$ & $15,5 \pm 8,46$ & $12,5 \pm 7,18$ \\
\hline & GIII & $26,83 \pm 15,55$ & $32,67 \pm 15,33$ & $16,17 \pm 7,41$ & $5,5 \pm 5,54$ & $5,67 \pm 6,83$ & $8,33 \pm 7,79$ & $12,5 \pm 14,22$ \\
\hline
\end{tabular}

T95= $=15$ da indução; $T 45=15$ minutos apos a indução; $T 75=30$ minutos após a indução; $180=5$ minutos após a infusão continua

Segundo Hvidberg et al. (1981), Cunnigham e Barry (1986) e Murphy (1985), alterações na $\mathrm{PaCO}_{2}$ são acompanhadas por alterações semelhantes na PIO e na PVC, podendo-se concluir que o aumento da PIO, após elevação da $\mathrm{ETCO}_{2}$, ocorre como resultado da vasodilatação coroidal ou da elevação da PVC ou, provavelmente, pela combinação de ambas. A importância da PVC sobre a PIO é bem conhecida. A elevação na PVC inibe a drenagem sangüínea do olho, resultando em aumento da PIO (Brunson, 1980; Murphy, 1985). Macri (1961b) sugeriu que alterações na PVC resultavam em alterações correspondentes no diâmetro dos vasos sangüíneos intra-oculares, mais provavelmente nos vasos dos processos ciliares. Afirmou, também, que alterações no diâmetro dos vasos influenciam a PIO, devido aos efeitos sobre o volume intra-ocular. Contudo, no presente estudo, os valores de PVC mantiveram-se dentro da normalidade durante todos os períodos, podendo-se constatar que, mesmo com a hipovolemia, a pré-carga foi mantida.

A PAM relacionou-se, indiretamente, com a $\mathrm{ETCO}_{2}$, pois seus valores continuaram em queda, enquanto que os da $\mathrm{ETCO}_{2}$ aumentaram. Os valores decrescentes de $\mathrm{ETCO}_{2}$, em T45, demonstraram a tentativa compensatória de oxigenação sangüínea por meio do aumento da FR. Os valores crescentes de $\mathrm{ETCO}_{2}$ correlacionaram-se, diretamente, com os valores da PIO. Duncalf e Weitzner (1963) e Brunson 
(1980) já haviam relatado que o aumento da pressão arterial de $\mathrm{CO}_{2}\left(\mathrm{PaCO}_{2}\right)$ elevava a PIO. As tensões de $\mathrm{CO}_{2}$ e de $\mathrm{O}_{2}$ no sangue arterial podem afetar a PIO por meio de três mecanismos: produção de HA, vasodilatação intra ou extra-ocular e alteração no tônus dos músculos extra-oculares (Duncalf e Weitzner, 1963; Cunnigham e Barry, 1986).

O desflurano diminui a função sistólica e diastólica do ventrículo esquerdo, além de diminuir a resistência vascular sistêmica e a pressão arterial, num padrão dependente da dose (Mcmurphy e Hodgson, 1996). Verificou-se essa ocorrência pelo discreto declínio nos valores da PAM, após o uso do desflurano. Anestésicos inalatórios, como halotano e isoflurano, diminuem a PIO por meio da diminuição da formação do HA e do aumento da drenagem trabecular (Artru, 1995; Sator et al., 1998). Em cães, o desflurano foi associado a valores baixos da PIO (Artru, 1995). Neste estudo, não foram observadas alterações na PIO, com o uso do desflurano.

Schutten e Van Horn (1977), Antal et al. (1978) e Bar-Ilan e Pessah (1986) observaram que a utilização de cetamina na anestesia de coelhos elevou a PIO. Cugini et al. (1997) afirmaram que a cetamina não possui efeito significativo sobre a PIO em pacientes humanos e pré-medicados. Na espécie eqüina, Trim et al. (1985) observaram que a combinação da cetamina com a xilazina e o bloqueio aurículo-palpebral não aumentavam significativamente a PIO. Jia et al. (2000) mediram a $\mathrm{PIO}$, em ratos, com a obstrução experimental da drenagem do HA, utilizando isoflurano ou cetamina, ou uma mistura de cetamina, xilazina e acepromazina. Os autores concluíram que todos esses protocolos anestésicos causaram rápido e substancial declínio na PIO. No presente estudo, foi observada discreta influência da cetamina sobre a PIO em todos os grupos. Segundo Peuler et al. (1975), o efeito da cetamina sobre a PIO pode ocorrer via sistema nervoso simpático e pela liberação de norepinefrina. Os autores sugeriram que o hipotálamo pode influenciar a PIO por meio de impulsos aferentes levados pelos nervos ciliares longos, e que a estimulação de muitos pontos pode provocar aumento ou redução da PIO, independente das alterações vasculares. Acrescentam, ainda, que as reduções nos valores da PIO são, provavelmente, mediadas pelo sistema nervoso simpático, pois podem ser evitadas quando o tronco simpático é seccionado. Essa hipótese talvez possa explicar a discreta diminuição da PIO logo após o início da infusão da cetamina.

$\mathrm{Na}$ forma como foi realizado este estudo, permite-se concluir que não houve correlação direta entre alterações da PAM e da PVC e a PIO; o desflurano não influenciou os resultados da PIO, porém observou-se discreta ação da cetamina em todos os grupos analisados e foi possível estabelecer relação entre valores de PIO e de $\mathrm{ETCO}_{2}$.

\section{AGRADECIMENTOS}

Ao Programa de Pós-graduação em Cirurgia Veterinária da FCAV/UNESP - Jaboticabal, SP, pelo apoio financeiro concedido e ao Professor Adriano Bonfim Carregaro, docente da Universidade de Franca - UNIFRAN, pelo auxílio com as análises estatísticas.

\section{REFERÊNCIAS BIBLIOGRÁFICAS}

ANTAL, M.; MUCSI, G.; FALUDI, A. Ketamine anesthesia and intraocular pressure. Annais Ophthalmol., v.10, p.1281-1284, 1978.

ARTRU, A.A. Rate of anterior chamber aqueous formation, trabecular outflow facility, and intraocular compliance during desflurane ou halothane anesthesia in dogs. Anesth. Analg., v.81, p.585-590, 1995.

BAR-ILAN, A.; PESSAH, N.I. On the use of ketamine in ocular phamacological studies. $J$. Ocular Pharmacol., v.2, p.335-344, 1986.

BRUNSON, D.B. Anesthesia in ophthalmic surgery. Vet. Clin. North Am.: Small Anim. Pract., v.10, p.481-495, 1980.

CLARKE, K.W.; SONG, D.Y.; LEE, Y.H. et al. Desflurane anaesthesia in the horse: minimum alveolar concentration following induction of anaesthesia with xylazine and ketamine. J. Vet. Anaesth., v.23, p.56-59, 1996.

COLLINS, B.K.; GROSS, M.E.; MOORE, C.P. et al. Physiologic, pharmacologic, and practical considerations for anesthesia of domestic 
animals with eye disease. J. Am. Vet. Med. Assoc., v.207, p.220-230, 1995.

CUGINI, U.; LANZETTA, P.; NADBATH, P. et al. Sedation with ketamine during cataract surgery. J. Catar. Refract. Surg., v.23, p.784786, 1997.

CUNNIGHAM, A.J.; BARRY, P. Rewiew article: Intraocular pressure - physiology and implications for anaesthetic management. Can. Anaesth. Soc. J., v.33, p.195-208, 1986.

CUNNIGHAM, J.G. Tratado de fisiologia veterinária. Rio de Janeiro: Guanabara Koogan, 1993. 454p.

DUNCALF, D.; WEITZNER, W.S. The influence of ventilation and hypercapnea on intraocular pressure during anesthesia. Anesth. Analg., v.42, p.232-246, 1963.

HOEL, P.G. Elementary statistics. 3.ed. New York: John Wiley \& Sons, 1974. 368p.

HVIDBERG, A.; KESSING, SV. V.; FERNANDES, A. Effect of changes in $\mathrm{PCO}_{2}$ and body positions on intraocular pressure during general anesthesia. Acta Ophthalmol., v.59, p.465-475, 1981

JANTZEN, J.P.; HENNES, H.J.; ROCKELS, R. et al. Deliberate arterial hypotension does not reduce intraocular pressure in pigs. Anesthesiology, v.77, p.536-540, 1992.

JIA, L.; CEPURNA, W.C.; JOHNSON, E.C. et al. Effect of general anesthetics on IOP in rats with experimental aqueous outflow obstrution. Invest. Ophthalmol. Vis. Sci., v.41, p.3415-3419, 2000.

MACRI, F.J. Interdependence of venous and eye pressure. Arch. Ophthalmol., v.65, p.150-7, 1961b.

MACRI, F.J. Vascular pressure relationship and the intraocular pressure. Arch. Ophthalmol., v.65, p.133-6, 1961a.
MASSONE, F. Neuroleptoanalgesia e anestesia dissociativa. In: Anestesiologia Veterinária. 2.ed., Rio de Janeiro: Guanabara Koogan, 1994. p.92-97.

McMURPHY, R.M.; HODGSON, D.S. Cardiopulmonary effects os desflurane in cats. Am. J. Vet. Res., v.57, p.376-370, 1996.

McMURPHY, R.M.; HODGSON, D.S. The minimum alveolar concentration of desflurane in cats. Vet. Surg., v.24, p.453-455, 1995.

MICHELSON, G.; GROH, M.; GRÜNDLER, A. Regulation of ocular blood flow during increases of arterial blood pressure. Br. J. Ophthalmol., v.78, p.461-465, 1994.

MURPHY, D.F. Anesthesia and intraocular pressure. Anesth. Analg., v.64, p.520-530, 1985.

NUNES, N. Monitoração da anestesia. In: FANTONI, D.T.; CORTOPASSI, S.R. Anestesia em cães e gatos. São Paulo: Roca, 2002. p.64-81.

NUNES, N.; LAUS, J.L. Técnicas anestésicas destinadas à cirurgia ocular no cão. $B r . J$. Vet. Res. Anim. Sci., v.32, p.177-180, 1995.

PEULER, M.; GLASS, D.D.; ARENS, J.F. Ketamine and intraocular pressure. Anesthesiology, v.43, p.575-578, 1975.

SATOR, S.; WILDLING, E.; SCHABERNIG, C. et al. Desflurane maintains intraocular pressure at an equivalent level to isoflurane and propofol during unstressed non-ophthalmic surgery. Br. J. Anaesth., v.80, p.243-244, 1998.

SCHUTTEN, W.H.; VAN HORN, D.L. The effects of ketamine sedation and ketaminepentobarbital anesthesia upon the intraocular pressure of the rabbit. Invest. Ophthalmol. Vis. Sci., v.16, p.531-534, 1977.

TRIM, C.M.; COLBERN, G.T.; MARTIN, C.L. Effect of xilazina and ketamine on intraocular pressure in horses. Vet. Rec., v.117, p.442-443, 1985. 